\title{
Die neue HIN Plattform - bereit für die Zukunft
}

Thomas Gwender

Korrespondenz:

Sabine Spengler

Health Info Net AG

Pflanzschulstrasse 3

CH-8400 Winterthur

Tel. 0522350270

Fax 0522350272

andreas.nievergelt@hin.ch
Wie migrieren wir über 13000 Einzelkunden und 200 Institutionen von einer alten auf eine neue Sicherheitsplattform, ohne das Tagesgeschäft unserer Kundinnen und Kunden zu stark zu beeinträchtigen? Sicher eine der wichtigsten Fragen, mit denen das Projektteam bei der Umsetzung der neuen HIN Plattform konfrontiert wurde. Zum Zeitpunkt, als diese Frage auch beantwortet werden musste, lagen bereits zwei Jahre intensiver Projektarbeit hinter dem Team.

\section{Mangelnde Bedürfniserfüllung und einge- schränkte Funktionalität}

Bereits 2007 wurde erkannt, dass mit der aktuellen Architektur und der eingesetzten Technologie die zukünftigen Bedürfnisse der Nutzerinnen und Nutzer und die Anforderungen aus der eHealth-Bewegung der Schweiz nicht erfüllt werden können. Unter der Leitung des Management Teams der BlueCare wurde die Konzeptphase zum Aufbau der neuen HIN Plattform gestartet. Im Mai 2009 wurde die Freigabe für die Umsetzung durch den HIN Verwaltungsrat erteilt. Die Herkules-Arbeit konnte beginnen.

\section{Externe Überprüfung}

Aufgrund der grossen Investition entschied sich die FMH im Juli 2009, das Projekt durch eine neutrale externe Unternehmung überprüfen zu lassen. Die Analyse umfasste die strategische Ausrichtung der HIN AG, den Business Case und die technische Lösung der neuen HIN Plattform. Externe Reviews helfen, mögliche Schwachstellen zu erkennen und Korrekturmassnahmen einzuleiten. Dies war zum Glück nicht notwendig. Das Resultat der Analyse war positiv, und die Empfehlungen zu Veränderungen konzentrierten sich auf Anpassungen der damals aktuellen Strategie und auf eine mögliche Veränderung des Aktionariats durch Aufnahme starker Partner.

\section{Erste neue Dienste: HIN-Secure-Webmail - standortunabhängiger Zugang}

Anfang September 2010 konnten die ersten Dienste basierend auf der neuen Infrastruktur in Betrieb genommen werden. Es wurden zwei lange pendente Bedürfnisse der Ärzteschaft erfüllt: HIN Webmail und der Zugang zur HIN Plattform ohne ASAS Software wurden lanciert. Seither kann «anytime - anywhere» auf HIN zugegriffen werden. Die HIN Mailbox oder der Praxisspiegel der TrustCenter und alle anderen HIN Dienste sind heute ab beinahe jedem Gerät mit Internet-Anschluss nutzbar. Der Zugang über SMS Code (TAN) ist dabei klar favorisiert, aber auch der Einsatz der FMH HPC und der SuisseID wird genutzt.
Knapp 3000 HIN Kundinnen und Kunden nutzen heute bereits diese alternativen Zugangsarten und Secure Webmail.

\section{Abschluss der Umsetzung}

Im Dezember 2010 war der grösste Teil der technischen Umsetzung abgeschlossen. Die neue HIN Plattform war bereit für den Start. Innerhalb von $20 \mathrm{Mo}-$ naten wurde die neue Architektur unter der Gesamtverantwortung der BlueCare umgesetzt. Die wichtigsten neuen Komponenten sind:

- Identity und Access Management System von Oracle;

- Public Key Infrastruktur (PKI) vom Schweizer Provider QuoVadis;

- Secure Mail Infrastruktur vom Schweizer Hersteller SEPPmail;

- von Zühlke Engineering AG entwickelter Java basierter HIN Client.

Die Vorgabe, Standardkomponenten und Standardtechnologien einzusetzen, wurde rigoros verfolgt, und das Ergebnis bestätigte dieses Vorgehen. Die geplanten Funktionalitäten wurden umgesetzt und das Budget bis zum Start der Migration nur um $5 \%$ überschritten.

\section{März 2011: der Start der Migration}

Wie migrieren wir über 13000 Einzelkunden und 200 Institutionen von einer alten auf eine neue Sicherheitsplattform? Diese Frage wurde natürlich bereits früher aktuell. Für Ärztinnen und Ärzte heisst die Migration die Installation des neuen HIN Clients. Im Hintergrund wird dadurch eine Umschaltung von der alten ASAS Plattform auf die neue HIN Plattform durchgeführt. Gleichzeitig wird das der HIN Identität hinterlegte Zertifikat durch eHealth Suisse kompatible X.509 Zertifikate von QuoVadis ersetzt.

Dasselbe Vorgehen wird auch bei den institutionellen Kunden angewendet. HIN-MailgatewayKunden - so zum Beispiel über 150 Spitäler, total knapp 150 Domänen - müssen auf eine neue Version der HIN-Mailgateway-Software migrieren und nutzen so in Zukunft ebenfalls die neue HIN Plattform.

Die Migration der 13000 HIN Abonnenten startete am 2. März 2011 nach einer fünfmonatigen Testphase. Lernvideos, detaillierte Anleitungen für Mac und Windows und natürlich das HIN-Support-Team stand bereit. Die Kundinnen und Kunden wurden per Mail in Tranchen zur Migration eingeladen. Bis Anfang Juli 2011 sind über 9000 dieser Einladung gefolgt. Das erste Fazit nach den ersten Monaten: Ver- 


\section{Abbildung 1}

Die HIN Plattform und die angeschlossenen Akteure. 200 Institutionen und über 13000 Leistungserbringer sind vernetzt. Die breite und skalierbare HIN-Secure-Mail-Produktpalette vom Einzelplatzsystem bis zum Secure Mailgateway mit unlimitierter Anzahl gesicherter Mailboxen deckt jedes Bedürfnis ab.

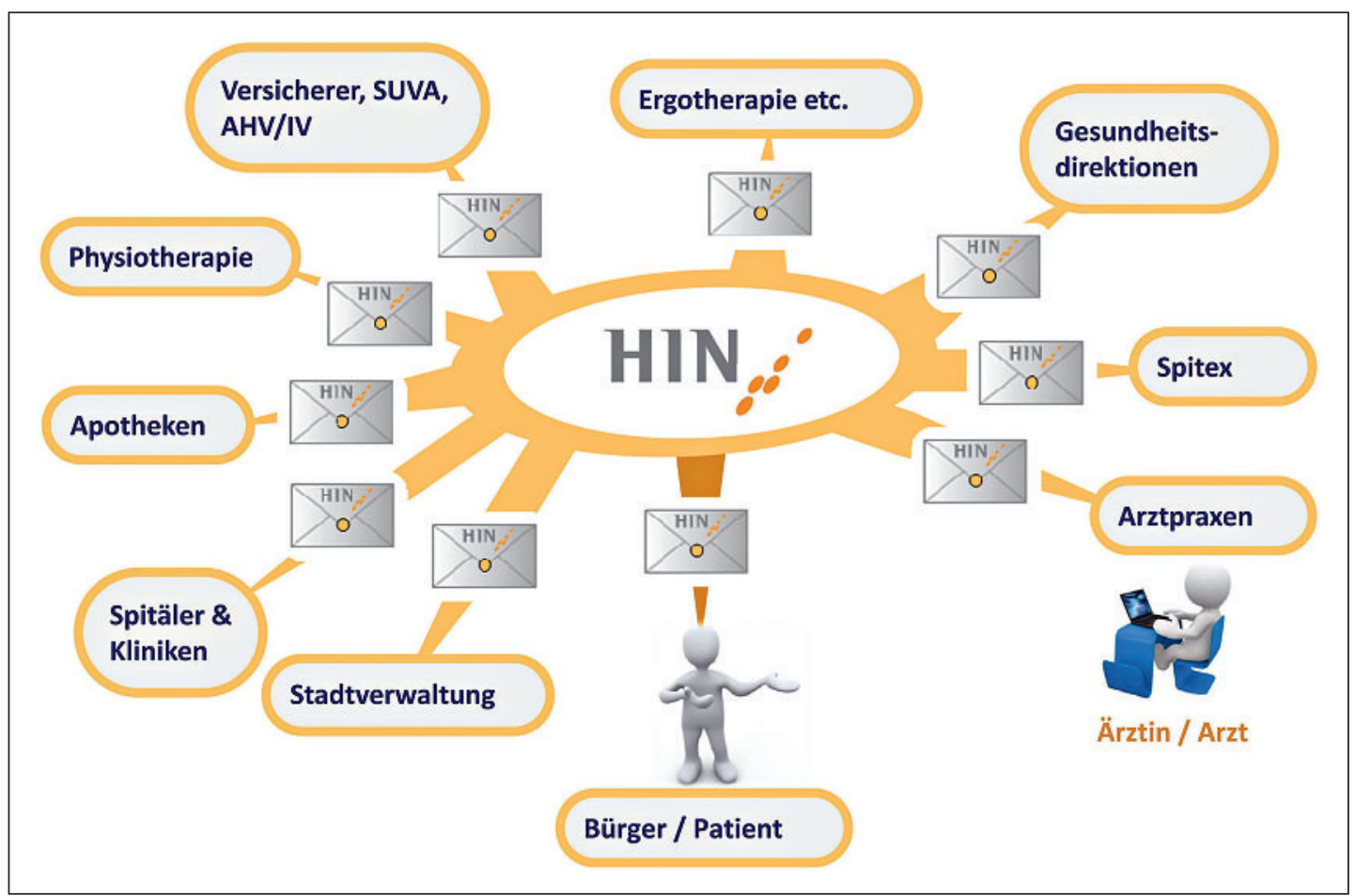

glichen mit der Komplexität des Vorhabens, fielen die aufgetretenen Schwierigkeiten insgesamt gering aus.

\section{Adieu ASAS - willkommen Zukunft}

Die ASAS Plattform wird im August 2011 abgeschaltet. Über die letzten 10 Jahre leistete die ASAS Technologie gute Dienste und half eine Gemeinschaft aufzubauen, die wahrscheinlich weltweit einmalig ist. tigen Möglichkeiten sind aber enorm. So wird noch dieses Jahr die Funktion «Secure Mail an Patienten» aktiviert werden. Für Anbieter von Applikationen und eHealth-Anwendungen ist SAML das Zauberwort. SAML ermöglicht den sicheren Austausch von Authentifizierungs- und Autorisierungsinformationen über Organisationsgrenzen hinweg. Die HIN Identität kann somit für den Zugang zu verschiedens-

\section{«Einfache Nutzung von Sicherheitstechnologien ist auch heute noch ein Knackpunkt - ausser für die Schweizer Ärzteschaft.»}

Einfache Nutzung von Sicherheitstechnologien ist auch heute noch ein Knackpunkt - ausser für die Schweizer Ärzteschaft. Die rund 8 Millionen gesichert übertragenen HIN-E-Mails sind nur ein Beweis, dass hohe Sicherheit auch benutzerfreundlich sein kann.

Die neue Plattform ersetzt heute in erster Linie die Funktionalitäten der ASAS Plattform. Die zukünf- ten Diensten bis hin zu Patientendossiers genutzt werden - natürlich ohne zusätzliche Eingabe von User-Id und Passwort. HIN ist somit wirklich wieder bereit für die Zukunft. Die technischen Grundlagen sind geschaffen und die Position der Ärzteschaft in der heiklen Entwicklungsphase von eHealth in der Schweiz wurde gestärkt. Nutzen wir diese Position. 